\title{
A Cautionary Note on the Use of Split-YFP/BiFC in Plant Protein-Protein Interaction Studies
}

\author{
Anneke Horstman ${ }^{1}$, Isabella Antonia Nougalli Tonaco ${ }^{1}$, Kim Boutilier ${ }^{1}$ and \\ Richard G. H. Immink ${ }^{1,2, *}$
}

1 Plant Research International, Droevendaalsesteeg 1, 6708 PB Wageningen, The Netherlands; E-Mails: anneke.horstman@wur.nl (A.H.); inougalli@gmail.com (I.A.N.T.); kim.boutilier@wur.nl (K.B.)

2 Physiology of Flower Bulbs, Department of Plant Physiology, Wageningen University, Droevendaalsesteeg 1, 6708 PB Wageningen, The Netherlands

* Author to whom correspondence should be addressed; E-Mail: richard.immink@wur.nl; Tel.: +31-6-13168407; Fax: +31-317-418094.

Received: 3 April 2014; in revised form: 17 April 2014 / Accepted: 20 May 2014 /

Published: 30 May 2014

\begin{abstract}
Since its introduction in plants 10 years ago, the bimolecular fluorescence complementation (BiFC) method, or split-YFP (yellow fluorescent protein), has gained popularity within the plant biology field as a method to study protein-protein interactions. $\mathrm{BiFC}$ is based on the restoration of fluorescence after the two non-fluorescent halves of a fluorescent protein are brought together by a protein-protein interaction event. The major drawback of BiFC is that the fluorescent protein halves are prone to self-assembly independent of a protein-protein interaction event. To circumvent this problem, several modifications of the technique have been suggested, but these modifications have not lead to improvements in plant $\mathrm{BiFC}$ protocols. Therefore, it remains crucial to include appropriate internal controls. Our literature survey of recent BiFC studies in plants shows that most studies use inappropriate controls, and a qualitative rather than quantitative read-out of fluorescence. Therefore, we provide a cautionary note and beginner's guideline for the setup of BiFC experiments, discussing each step of the protocol, including vector choice, plant expression systems, negative controls, and signal detection. In addition, we present our experience with $\mathrm{BiFC}$ with respect to self-assembly, peptide linkers, and incubation temperature. With this note, we aim to provide a guideline that will improve the quality of plant $\mathrm{BiFC}$ experiments.
\end{abstract}


Keywords: protein-protein interaction; split-YFP (yellow fluorescent protein); bimolecular fluorescence complementation (BiFC); protein complementation assay (PCA); in planta; fluorescence microscopy

\section{Introduction}

The vast majority of proteins encoded by a genome function in multi-protein complexes [1]. Identifying these protein-protein interactions can provide insight into the functions of individual proteins, as well as the biological processes they control. A large variety of high-throughput technologies have been developed in the past 20 years to identify protein-protein interactions, including a toolbox of techniques to detect or confirm putative interactions in vivo under physiologically relevant conditions [1,2]. The bimolecular fluorescence complementation (BiFC) assay, also referred to as "split-fluorescent protein" technology (e.g., split-YFP), is one of the most popular and frequently used methods in the plant field to study protein-protein interactions in vivo (reviewed in [3]). BiFC is based on the in vivo reconstitution of fluorescence after two non-fluorescent halves of a fluorescent protein (FP) are brought together by a protein-protein interaction event (Figure 1). As such, BiFC not only provides information on whether two proteins interact, but can also be used to determine the cellular and sub-cellular site of a protein-protein interaction event. The possibility to split a FP into two halves and to use these for the detection of interactions between molecules was first described in 2000 for the GREEN FLUORESCENT PROTEIN (GFP) [4]. Shortly thereafter, this method was used to detect in vivo protein-protein interactions in COS-1, NIH3T3, and HeLa cells [5], and later in plants [6,7]. The ease of implementation of the technology without the need for sophisticated equipment to detect the fluorescence signal has made BiFC a popular technology. In many cases, BiFC is the first method of choice for testing potential protein-protein interactions in planta and to confirm the outcomes of large-scale yeast-based or in vitro protein-protein interaction studies. The popularity of BiFC inspired researchers to optimize and modify the method to make it suitable for additional applications, including the development of multicolor BiFC for studying competition between interacting protein pairs or to simultaneously visualize multiple interactions in the same cell [8-10], and BiFC-FRET (Fluorescence Resonance Energy Transfer) for the detection of higher-order protein complex formation [11,12]. In the majority of plant studies, the split YFP-tagged proteins are overexpressed transiently or stably in isolated cells (protoplasts) or cell cultures; however, the BiFC method was recently used to study protein-protein interactions in intact plant tissues using native promoters to drive expression of the tagged proteins [13]. 
Figure 1. Schematic representation of the split-YFP (yellow fluorescent protein)/BiFC (bimolecular fluorescence complementation) method based on the YFP. YFP is split into two non-fluorescent "halves", an $N$-terminal part/half of the protein $\left(\mathrm{YFP}^{\mathrm{N}}\right)$ and a $C$-terminal part/half of the protein $\left(\mathrm{YFP}^{\mathrm{C}}\right)$, which are then fused to the proteins of interest (P1 and P2). The YFP molecule is reconstituted upon interaction between P1 and P2, resulting in yellow fluorescence when the molecules are excited with the correct wavelength.
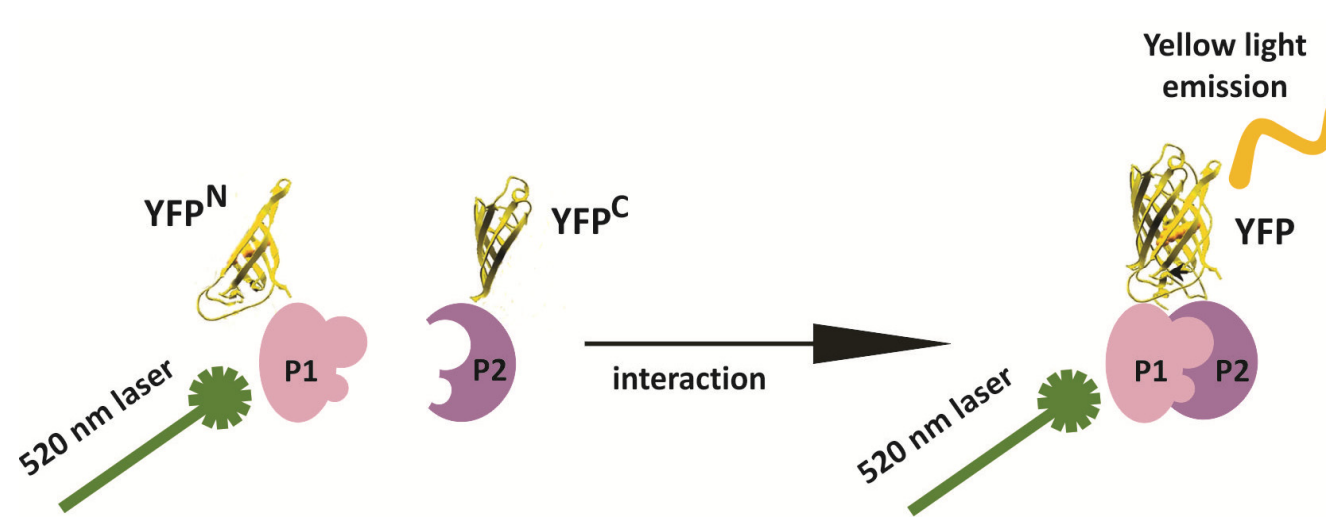

Despite its widespread use, the BiFC method does have a number of shortcomings for the detection and visualization of protein-protein interactions. The major drawback of the system is the ability of the two FP halves to reassemble in the absence of a bona fide protein-protein interaction. This so-called "self-assembly" of the FP halves can result in a high background signal, leading to a false-positive BiFC signal for a protein-protein interaction. To address this and other problems, a myriad of technical modifications have been implemented, including changing the split position in eYFP or YFP Venus from amino acid (AA) 155 to AA173 or AA210 [14], introduction of point mutations to suppress self-assembly of the two FP halves [15,16], and the use of negative controls, including point-mutated versions of the proteins under study [17]. Based on these observations, standard protocols have been developed [3,17,18] and additional optimization steps have been proposed to generate a more reliable and robust assay. Unfortunately, none of the proposed changes to improve the robustness and reliability of the method appear to be generally applicable in plants [3]. Similarly, improvements developed for a mammalian expression system did not result in a more reliable read-out in Xenopus [19].

We performed an inventory of the recent literature in the plant BiFC field and conclude that despite the awareness of shortcomings in the BiFC technology, the majority of researchers fail to include the correct internal controls and also incorrectly evaluate the results. Therefore, we present a guideline for BiFC use in plants, highlighting the most critical steps in the protocol and providing practical considerations for each individual step.

\section{Results and Discussion}

\subsection{Inventory of BiFC (Bimolecular Fluorescence Complementation) Use in Plant Studies}

Ten years ago, the first publications appeared showing the potential of the BiFC method for the detection and confirmation of protein-protein interactions in living plant cells [6,7]. Since then, a range of novel vectors and proposed improved protocols, mainly based on studies in mammalian cells, has been developed, with the goal to reduce the false-discovery rate and to improve the robustness of 
this technique. We performed a literature survey to determine which vectors, internal controls, and expression systems are used by the plant scientific community. A PubMed [20] search was performed in February 2014 using the terms "BiFC" and "plant" and the 100 most recent experimental papers were selected for analysis. From these studies, we extracted information about how the BiFC assay was performed (Table S1). Analysis of this dataset revealed that the majority of recent BiFC studies were carried out using the original vectors or using home-made vectors with the split position in the YFP molecule around amino acid 155 (AA155) [6,7]. Our analysis revealed that the newly developed vectors for the mammalian field have not been implemented in the plant field. One of the reasons might be that for plant systems, these new vectors do not solve the problem of the high false-discovery rate, as discussed below in Section 2.2.1. Remarkably and more problematic, the majority of BiFC experiments were conducted without any or with inappropriate internal controls (Figure 2A, see discussion below in Section 2.2.3). Furthermore, we noticed that in more than $90 \%$ of the studies only qualitative measurements of fluorescence signal were performed (Figure 2B, see further discussion below in Section 2.2.5).

Figure 2. Survey of experimental set-up used in plant BiFC experiments. (A) Negative controls used in recent BiFC experiments. The suitability of a control is scaled using shading from dark red (the worst) to dark green (the best). The red classes indicate controls with a higher incidence of detecting false positives. The green classes represent suitable controls. The yellow class indicates a control of intermediate quality. Schematic representations of the controls are shown to the right. $\mathrm{P} 1$ and $\mathrm{P} 2$ represent the two proteins of interest, PX and PY indicate proteins that are related and unrelated, respectively to the protein of interest, and $\mathrm{mP1}$ represents a mutant or truncated version of $\mathrm{P} 1$; (B) Percentage of $\mathrm{BiFC}$ experiments in which a qualitative (red) or quantitative (green) read-out of the fluorescence signal was measured.

A
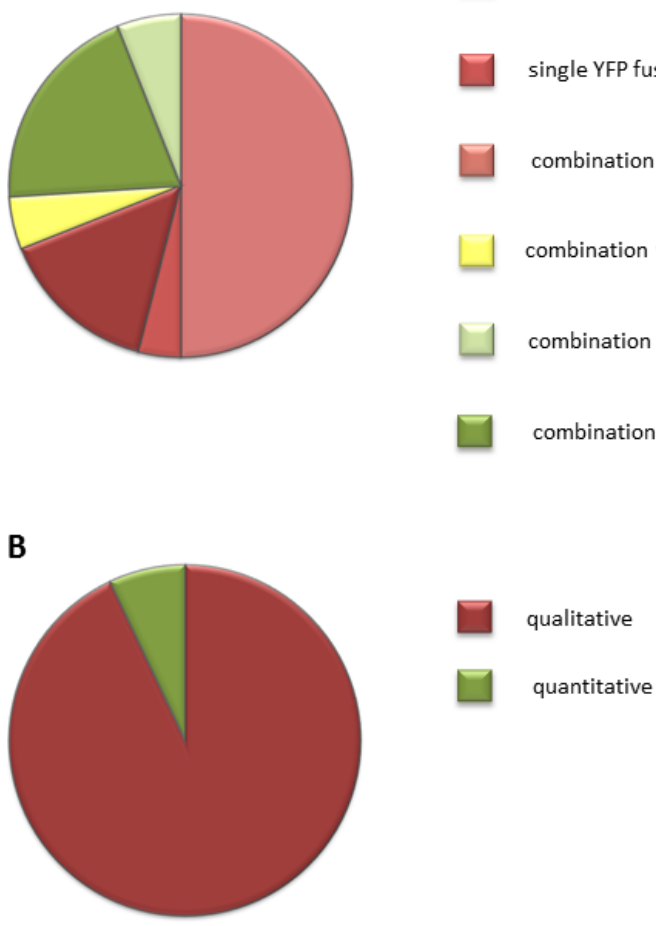

no or not clearly specified control

single YFP fusion

combination with unfused YFP half

combination with unrelated protein

combination with related protein

combination with mutated protein

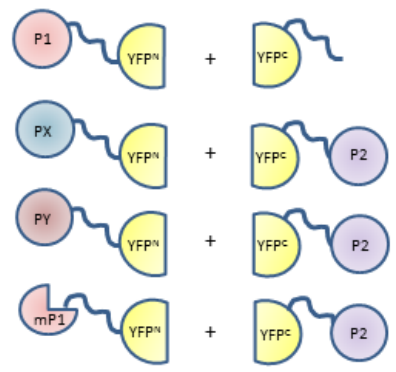

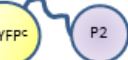

YFpc

YFPC $P 2$ 
Based on this survey, we conclude that the BiFC method is generally not executed in the proper manner. We therefore provide a guideline that can be followed for the design of an optimal BiFC experiment. This guideline is not meant to replace existing protocols (e.g., $[3,17,18]$ ), but rather, to provide additional information and notes on critical points in the method, based on published experiments and unpublished studies from our lab. This guideline will help the plant community to perform high quality BiFC studies, with an ensuing improvement in the quality of protein-protein interaction data.

\subsection{Overview of the BiFC Method}

An overview of the BiFC method is presented in Figure 3. Prior to the start of a BiFC experiment, a number of choices have to be made, including the selection of vectors and negative and positive controls, and the expression system, each of which influences the outcome and quality of the experiment. In this section, we discuss the most important considerations for each individual step of the BiFC protocol.

Figure 3. Flow diagram representing the steps and critical points in a BiFC experiment.

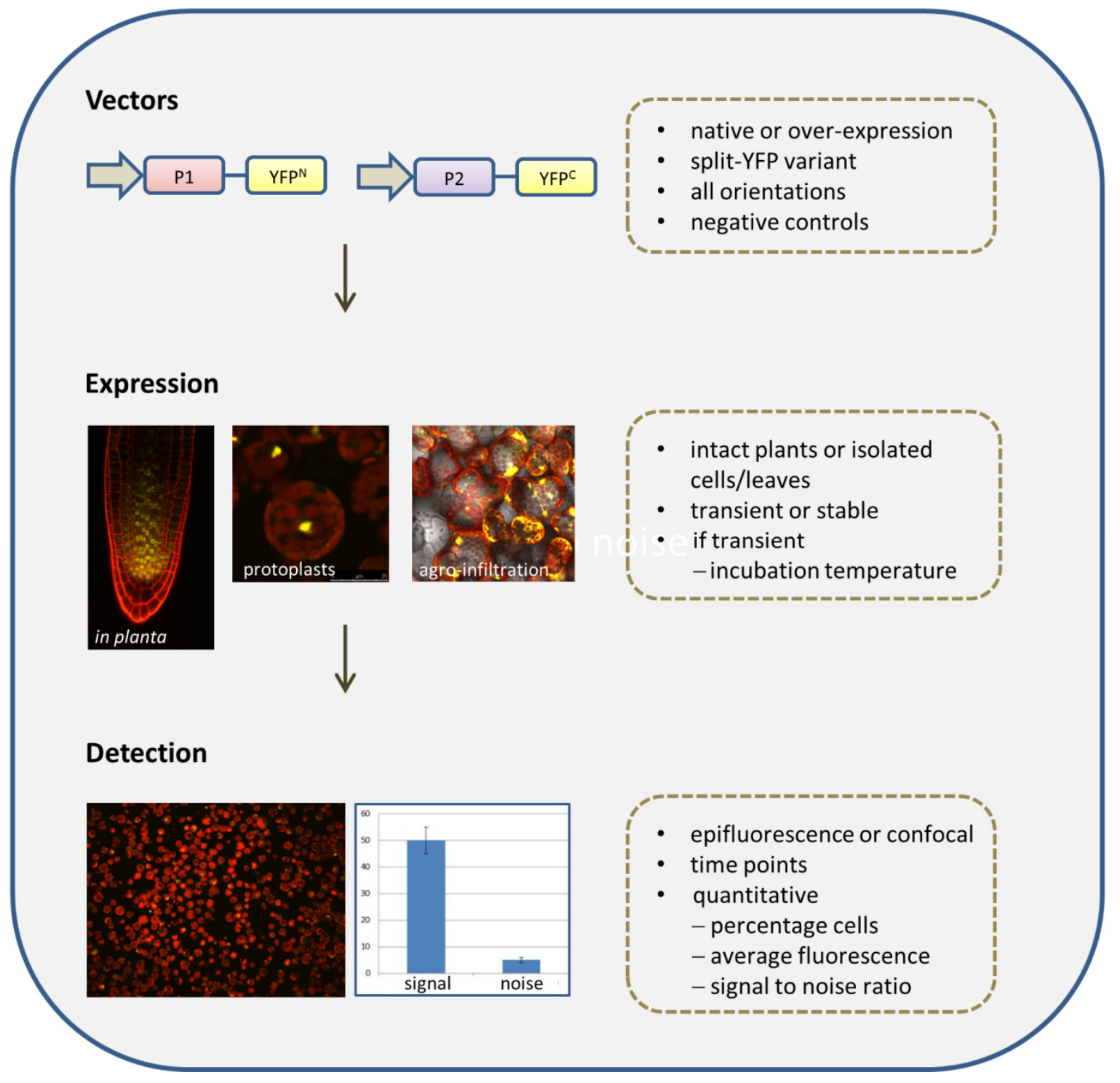




\subsubsection{Selection of Vectors}

When the BiFC method was introduced in plants in 2004, a small number of vector sets were available that all split eYFP in the loop between the seventh and eighth $\beta$-sheet (around AA155), and that expressed the fusion proteins from the strong constitutive Cauliflower Mosaic Virus 35S RNA promoter (CaMV35S) [6,7]. Unfortunately, this split position promotes irreversible self-interaction capacity of the two non-fluorescent fragments, which may result in the detection of false positive protein-protein interactions if inappropriate controls are used. A few years later, new vectors were developed that split the YFP between $\beta$-sheet nine and ten (AA173), and the resulting $\mathrm{YFP}^{\mathrm{N}}(1-173)$ fragment was combined with $\mathrm{YFP}^{\mathrm{C}}$ (156-239) [10]. This novel split position and combination of YFP fragments resulted in an increased signal for a positive control protein-protein interaction. However, the signal due to self-assembly also increased, which did not improve the signal (tested interaction)-to-noise (self-assembly) ratio [10]. Hence, this novel split position and combination of YFP fragments did not circumvent the problem of self-assembly and the accompanying high fluorescence signal. Aiming to minimize self-assembly, point mutations were introduced in the YFP halves, but with limited and varying success. Of all the reported mutations [3], only the amino acid change I152L in the Venus YFP protein seems to give a consistent better signal-to-noise ratio when used in animal cells [15]. However, in plant cells, this change results in a very weak fluorescence signal even with strongly interacting proteins [21].

It has also been proposed that the sequence and length of the peptide linker between the protein of interest and the YFP fragment could influence the complementation capacity of the split YFP fragments by affecting the flexibility and/or folding of the fused proteins, which in turn might be required for complex formation [22]. We tested three different vector sets varying substantially in the sequence and length of the peptide linker (Table 1) and obtained similar fluorescence complementation signals for the interacting petunia MADS (MCM1, AGAMOUS, DEFICIENS and Serum Response Factor) domain transcription factor proteins FLOWERING BINDING PROTEIN2 (FBP2) and FBP11. Our results suggest that this specific protein-protein interaction is not influenced by the characteristics of the peptide linker, but we cannot exclude that the peptide linker is of importance for proper folding and detection of interactions of other fusion proteins. Nonetheless, the observation that peptide linkers in commonly used BiFC vectors vary substantially, but still allow BiFC (see Table S1), suggests that the peptide linker sequence is not a critical factor for the success of a BiFC experiment.

In conclusion, our results and survey of the plant BiFC literature suggests that there is no evidence for the superiority of a particular BiFC vector set. Rather, it appears that reconstruction of the FP halves through protein-protein interaction depends more on the characteristics of the fused proteins than on the sequence of the YFP halves, the linker region, or the vector. Furthermore, BiFC efficiency differences have been observed between species, indicating that the cell type and the accompanying incubation conditions have a larger effect on BiFC than the vector itself. As discussed below, incorporating proper negative controls and experimental conditions seems to be of more importance for the success of a BIFC experiment. 
Table 1. Overview of BiFC constructs with different peptide linker sequences between the coding regions of the YFP halves and the coding regions of two interacting petunia MADS (MCM1, AGAMOUS, DEFICIENS and Serum Response Factor) domain transcription factor proteins. The indicated linker lengths include amino acids (AA) encoded by parts of the multiple cloning site. Vector combinations were tested upon transient transfection of petunia protoplasts. All expression cassettes were embedded in a $p U C$ vector backbone. Peptide linker sequences: Gateway-based cloning linker sequence [23]; RSIAT/KQKVMNH [5]; myc and HA tag [7].

\begin{tabular}{ccccccc}
\hline $\begin{array}{c}\text { BiFC Vector } \\
\text { Set }\end{array}$ & \multirow{2}{*}{ Promoter } & $\begin{array}{c}\text { Protein of } \\
\text { Interest }\end{array}$ & $\begin{array}{c}\text { Peptide } \\
\text { Linker }\end{array}$ & $\begin{array}{c}\text { Length } \\
(\text { AA) }\end{array}$ & $\begin{array}{c}\text { YFP } \\
\text { Part }\end{array}$ & Terminator \\
\hline \multirow{2}{*}{$1[24]$} & & FBP2 & Gateway & 17 & YFP $^{\mathrm{N}}$ & \\
& & FBP11 & Gateway & 18 & YFP $^{\mathrm{C}}$ & \\
2 & \multirow{2}{*}{ CaMV35S } & FBP2 & RSIAT & 15 & YFP $^{\mathrm{N}}$ & \multirow{2}{*}{ NOS } \\
& & FBP11 & KQKVMNH & 17 & YFP $^{\mathrm{C}}$ & \\
$3[7]$ & & FBP2 & Myc-c tag & 26 & YFP $^{\mathrm{N}}$ & \\
& & FBP11 & HA-tag & 25 & YFP $^{\mathrm{C}}$ & \\
\hline
\end{tabular}

\subsubsection{Fusion Orientations}

One factor that influences the ability to detect protein-protein interactions in BiFC assays is the effect of the YFP fusion on the protein of interest. Protein-protein interactions are mediated by specific protein domains and fusing other (fluorescent) proteins to a protein of interest can interfere with the interaction capacity of these domains by steric hindrance or due to mis-folding [25]. In addition, the three dimensional structure of a protein complex can also inhibit the reconstitution of the FP by spatial restrictions. Bracha-Drori et al. [6] showed that the fusion orientation can affect the amount of BiFC signal. Therefore, to exclude false negative combinations, it is recommended to generate and test all of the eight combinations of constructs in which the $N$ - and $C$-terminal fragments of the FP are fused to the $N$ - and $C$-terminus of the proteins of interest (Figure 4A). The functionality of these fusion proteins can also be tested by genetic complementation, provided a mutant phenotype is available for the protein of interest. We believe that a single positive combination can provide sufficient proof of protein-protein interaction, as long as suitable negative controls are included and a correct experimental set-up is followed. 
Figure 4. Two key elements of a $\mathrm{BiFC}$ experiment: fusion orientation and controls. (A) Each of the two YFP halves $\left(\mathrm{YFP}^{\mathrm{N}}\right.$ and $\left.\mathrm{YFP}^{\mathrm{C}}\right)$ can be fused at either its $N$ - or $C$-terminus, with the protein of interest. Likewise, the protein of interest can also be fused to the YFP half via its $N$ - or $C$-terminus. This creates four possible YFP-protein combinations for each protein of interest and eight combinations that should be tested for each interaction pair; (B) In addition to the protein-protein interaction to be tested, (i) appropriate negative controls should be incorporated in a $\mathrm{BiFC}$ experiment; These negative controls include substitution for one of the protein of interest by (ii) a mutated protein $(\mathrm{mP} 1)$; (iii) a related protein $(\mathrm{PX})$ that does not interact; and (iv) an unrelated protein (PY) with the same subcellular localization. The stars indicate YFP fluorescence due to self-assembly (one star) and expression due to a bona fide interaction between the test proteins (three stars).

A

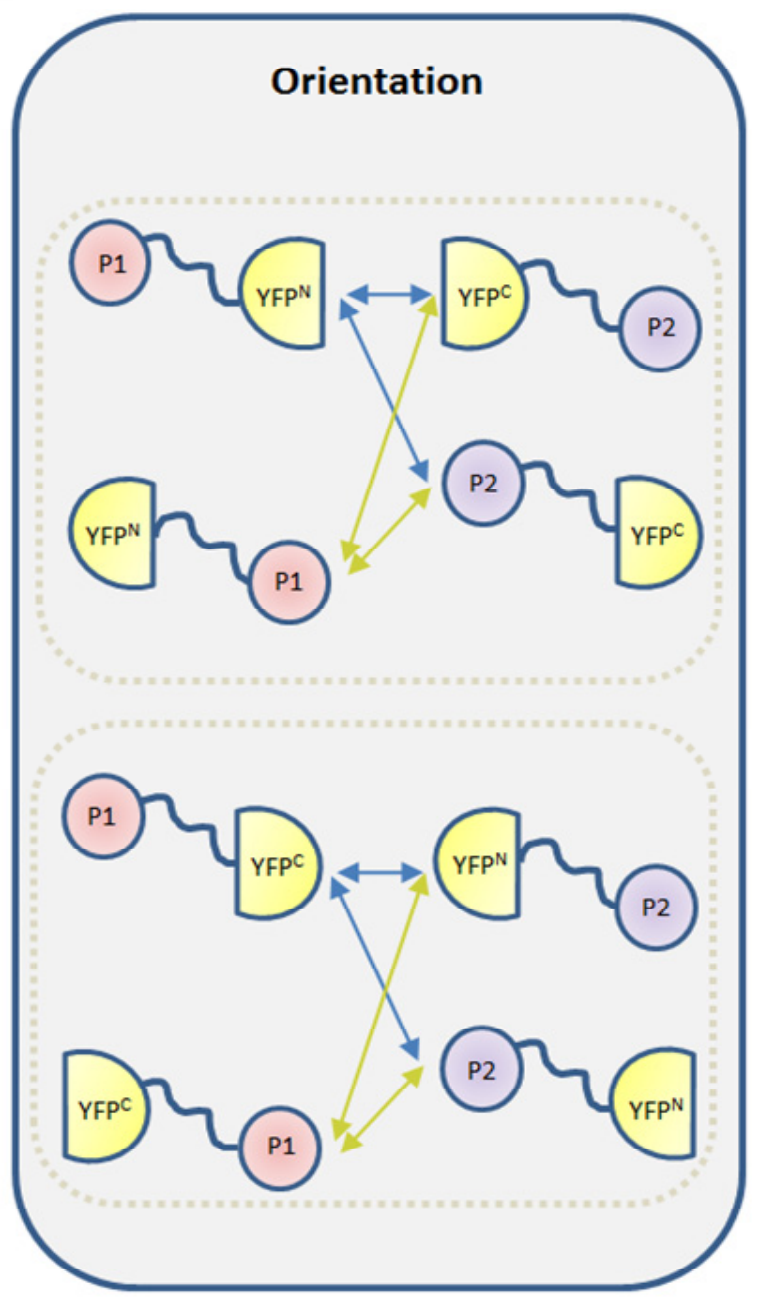

B

(i)

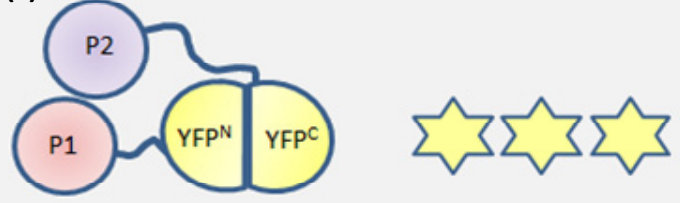

Controls

(ii)

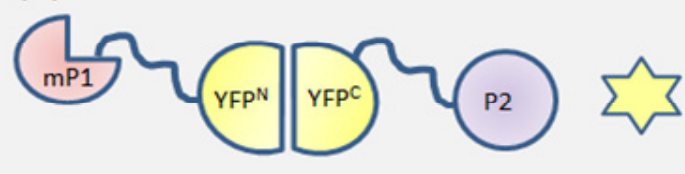

(iii)

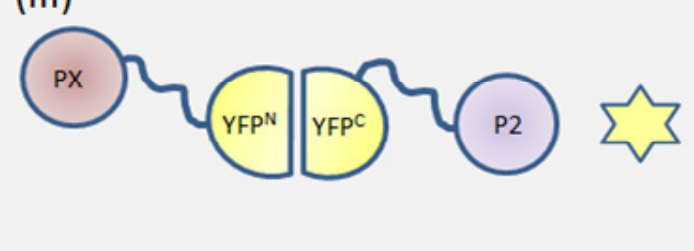

(iv)

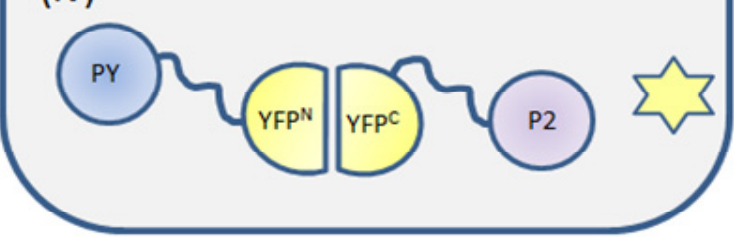

\subsubsection{Negative Controls}

The major disadvantage of $\mathrm{BiFC}$ as a method to detect protein-protein interactions is the signal that results from aspecific and irreversible interaction of the $N$ - and $C$-terminal parts of the FP in the absence of interaction between the fused proteins of interest. For this reason, choosing a proper negative control is a critical step in the design a BiFC experiment. The ideal negative control in a BiFC 
experiment is a translational fusion between one half of the FP and a truncated or mutated version of the protein of interest that is unable to bind to its interaction partner (Figure 4B; e.g., [26,27]). Development of this type of negative control implies that interaction domains or amino acids have been identified using an in vitro approach, such as yeast two-hybrid screening, using bioinformatics predictions or genetic complementation, and that the changes to the protein do not negatively affect its stability or folding. In lieu of this, a fusion with a protein that is related to the protein of interest, but that does not interact would be a good alternative (Figure 4B; e.g., [28,29]). If a non-interacting protein family member is not known or available, one could use an unrelated, non-interacting protein with the same cellular localisation (Figure 4B). We noticed that in a few cases in the literature, separate transfection with a plasmid comprising one protein of interest fused to $\mathrm{YFP}^{\mathrm{N}}$ or $\mathrm{YFP}^{\mathrm{C}}$ was used as a control (Table S1). However, this control does not report the aspecific interactions that might occur between split-YFP fragments, because only one of the two YFP fragments is expressed. Empty vector controls comprising either half of the split YFP molecule, but lacking the protein of interest, were used most commonly in combination with the expression of a protein of interest fused to the complementary YFP fragment. Although it has been suggested that the expression levels of these unfused non-fluorescent fragments is higher than the expression of fusion proteins [3], which would provide a conservative background level estimation, the subcellular localization of these split-YFP fragments might differ from that of the fusion between the split FP and protein of interest, thereby abolishing any potential for aspecific interaction and subsequent underestimation of the background. Note that the FP halves of negative controls should theoretically self-assemble, but may not do so due to interference by the fused test protein. It is therefore important to always use negative control that consistently exhibits a detectable fluorescence signal to obtain a conservative estimate of the signal-to-noise ratio.

To determine the background fluorescence levels caused by self-assembly of AA155-based BiFC vectors [24], we generated fusion constructs between TagRED FLUORESCENT PROTEIN (TagRFP) and either the $N$ - or the $C$-terminal YFP fragments $\left(\right.$ TagRFP-YFP $^{\mathrm{N}}$ and TagRFP-YFP ${ }^{\mathrm{C}}$ ). TagRFP is a monomeric fluorescent protein [30] and its use enabled us to confirm the expression of the individual fusion proteins. TagRFP expression was observed as early as eight hours after co-transfection of Arabidopsis protoplasts with single TagRFP fusion constructs or TagRFP-YFP ${ }^{\mathrm{C}}$ and $\mathrm{YFP}^{\mathrm{N}}-\mathrm{BBM}$, a fusion of $\mathrm{YFP}^{\mathrm{N}}$ with the transcription factor BABY BOOM (BBM; [31]). We noticed that in the double transfection, TagRFP fluorescence always coincided with weak YFP fluorescence. This fluorescence was not caused by bleed-through of TagRFP into the YFP channel, as no YFP signal was observed upon single transfections with TagRFP-YFP ${ }^{\mathrm{C}}$ or TagRFP-YFP ${ }^{\mathrm{N}}$ constructs alone (Figure 5). Since there is no indication that BBM and TagRFP proteins interact, this YFP signal likely reflects the signal from YFP self-assembly. Although the YFP signal is weak, this experiment shows that BiFC results should be interpreted with caution. BiFC experiments cannot be performed without a proper negative control: transfection with a single plasmid is not sufficient. Additionally, a quantitative read-out should be used to distinguish between a fluorescence complementation by a true protein-protein interaction and signal due to self-assembly of the two FP halves, which can be scored based on the inclusion of a suitable negative control. 
Figure 5. Self-assembly of YFP fragments. Confocal images of Arabidopsis protoplasts eight hours after transfection with either single TagRFP-YFP ${ }^{\mathrm{C}}$ or $-\mathrm{YFP}^{\mathrm{N}}$ plasmids, or with TagRFP-YFP ${ }^{\mathrm{C}}$ and $\mathrm{YFP}^{\mathrm{N}}$-BBM plasmid combinations. The first column shows an overlay image of the background autofluorescence signal (blue), the TagRFP signal (red) and the YFP signal (yellow). The second and third columns show only the TagRFP and YFP signals, respectively. Co-transfection of plasmids containing $\mathrm{YFP}^{\mathrm{N}}-\mathrm{BBM}$ and TagRFP-YFP fragments results in a weak YFP signal, showing the re-constitution of the YFP molecule in the absence of a protein-protein interaction (self-assembly), and indicating the need for suitable negative controls and a quantitative read-out of the fluorescent signal in BiFC experiments.

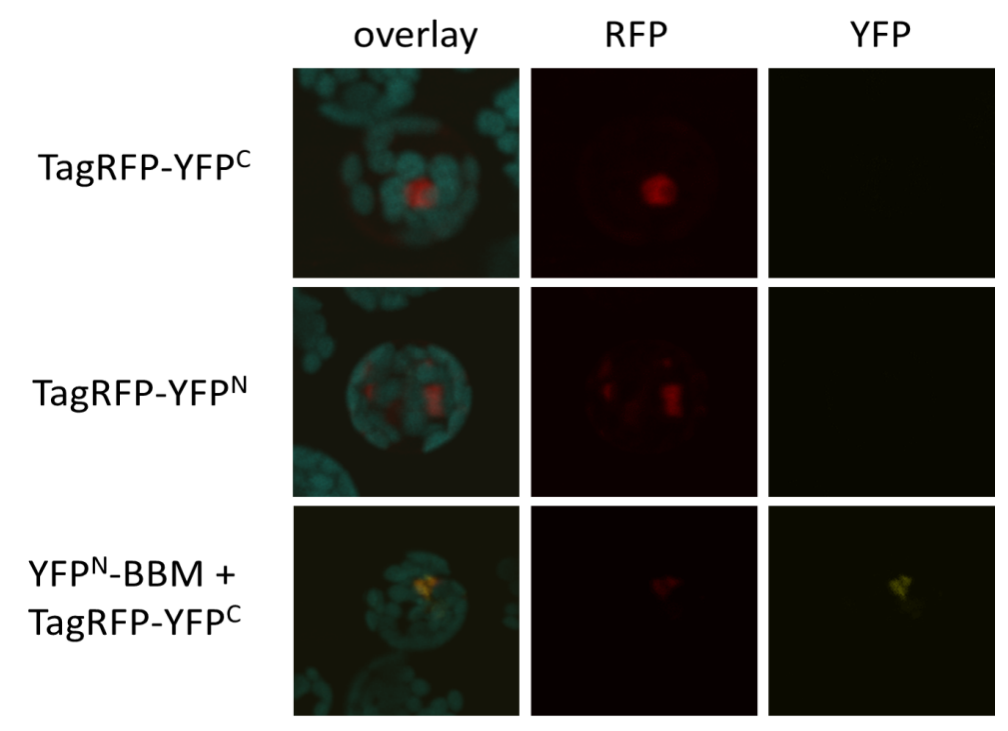

\subsubsection{The BiFC Assay: Expression Systems}

BiFC experiments in plants are almost exclusively carried out using transient expression systems, usually Agrobacterium infiltration of tobacco leaf cells or polyethylene glycol (PEG)-mediated transfection of leaf protoplasts. Several protocols have been published for both methods (e.g., [17,32-34]), and neither is considered superior. Alternatively, the BiFC assay can be performed in whole plants (in situ) using stable transformants in which the promoters of the protein of interest are used to drive expression of the YFP-fusion [13]. Studying protein-protein interactions in their native context ensures that any additional proteins that are required for a protein-protein interaction will be co-expressed in the correct tissue context, and that the quantitative relationship between the target protein and endogenous partner proteins is maintained. This approach can give insight about when and where a specific protein-protein interaction first occurs, however, the irreversibility of the re-assembly of the YFP halves excludes the possibility to study protein-protein interaction dynamics. Since the genomic integration site of a transgene can affect its expression, it is important to determine whether the expression level and tissue specificity/localization of the fusion protein corresponds to that of the endogenous gene, and that expression of the transgene does not confer any mutant phenotypes. Testing of multiple independent transgenic lines is therefore required. BiFC analyses in planta using native protein expression levels in the native cellular environment is the most elegant approach, but these type of studies are still in their infancy and need to be more thoroughly analyzed to determine their robustness and reliability. 
Another factor that needs to be considered in relation to the expression system is the culture temperature of the protoplasts or plant tissues. While performing BiFC experiments by transient transfection of petunia protoplasts, we noticed a strong negative effect of the culture temperature on fluorescence complementation. Petunia protoplasts are routinely cultured at $28{ }^{\circ} \mathrm{C}[35,36]$. A BiFC signal was not observed for the FBP2-FBP11 protein combination at this temperature, but at least $30 \%$ of the protoplasts showed a fluorescence signal when the protoplasts were incubated at $23{ }^{\circ} \mathrm{C}$. This difference is not due to misfolding of the petunia MADS domain proteins at $28{ }^{\circ} \mathrm{C}$, because strong fluorescence signals were obtained in protoplasts cultured at $28^{\circ} \mathrm{C}$ when the FBP2 and FBP11 proteins were tagged with a complete YFP fluorophore [36,37]. The high-temperature sensitivity therefore seems to be specifically associated with the BiFC method. In support of this, a four-hour pre-incubation of mammalian cells at lower temperatures prior to BiFC imaging significantly increases the fluorescence signal [38].

Regardless of the expression method used, it is important to determine whether the control proteins and the proteins of interest are expressed. This not only provides information about the level of protein expression, which can greatly influence the results, but also indicates if the fusion between the protein of interest-split YFP is intact.

\subsubsection{Detection Methods}

Fluorescence complementation in a BiFC experiment is usually detected using an epifluorescence microscope or a confocal laser scanning microscope (CLSM) (Supplementary Table S1). The qualitative analysis of $\mathrm{BiFC}$ experiments is problematic because of the self-assembly capacity of the two FP halves. Consequently, simply showing images of fluorescent cells from the protein combination of interest and non-fluorescent cells from a control transfection is insufficient proof of a protein-protein interaction. Rather, a quantitative comparison should be made between the signal obtained with the protein combination under study and the signal obtained with a proper negative control combination.

When using transient overexpression, it is important to realize that the irreversible nature of FP complementation leads to accumulation of the fluorescence signal in time, both for the tested interaction and the control experiment. The complementation of the FP by a protein-protein interaction and accumulation of fluorescence signal will proceed faster than the self-assembly in the control experiment due to the higher binding affinity of the interacting fusion proteins. Therefore, it is advisable to measure fluorescence signals at different time points after transfection, as saturation of fluorescence signals influences the signal-to-noise ratio. Unfortunately, the vast majority of BiFC experiments are performed in a qualitative manner (Figure 2; Table S1). One method for BiFC quantification is to determine the percentage of positive cells for both the controls and the tested protein-protein interaction. Alternatively, the signal (tested interaction)-to-noise (self-assembly signal) ratio can be determined by measuring fluorescence intensities. Signal intensities can be determined from fluorescence images, but this approach is time-consuming, as it requires measurement of many cells on a one-by-one basis to determine average signal intensity. A faster way to analyze average fluorescence intensity in a population of cells is by fluorometry or by flow cytometry [39]. Because the amount of expressed fusion proteins greatly influences the results, it is important to determine the expression levels of the different fusions within the population of cells by Western blotting. 
Subsequently, the BiFC signal intensity of the cell population can be normalized against the amount of fusion protein.

\section{Experimental Section}

\subsection{Test of Different Peptide Linker Sequences}

The effect of using different peptide linker sequences between the halves of YFP and the protein of interest was examined in petunia protoplasts. The interaction between the petunia MADS domain proteins FBP2 and FBP11 [36] was used as positive control. All vectors used for this experiment were based on the $p U C$ vector backbone and are described in Table 1. Isolation and transfection of petunia W115 leaf protoplasts was performed as described previously [35,36]. After transfection, protoplasts were incubated overnight in the dark at $28^{\circ} \mathrm{C}$ (according to the original protocol) or at $23{ }^{\circ} \mathrm{C}$.

\subsection{Self-Assembly Capacity of YFP (Yellow Fluorescent Protein) Halves}

$B B M$ [31] and TagRFP [30] cDNA entry clones (pDONR207) were used to generate the $Y F P^{N}-B B M$, TagRFP-YFP ${ }^{N}, T a g R F P-Y F P^{C}$ plasmids for Arabidopsis protoplast transfection. The plasmids were cloned using recombination into Gateway-compatible BiFC vectors [24]. Arabidopsis protoplast isolation followed the procedures described in [40], except that leaves of three to four week-old Col-0 seedlings were used. Protoplast transfections were carried out as described in [41], but with a transfection time of $10 \mathrm{~min}$. Fluorescence was viewed $8 \mathrm{~h}$ after transfection by CLSM.

\section{Conclusions}

Split-YFP/BiFC is a widely used method for the detection and confirmation of protein-protein interactions in living plant cells. Nevertheless, the usefulness of this technology is overshadowed by self-assembly of the two halves of the FP, which results in the detection of fluorescent signal regardless of an interaction between the proteins of interest. Consequently, the introduction of control experiments is essential to obtain evidence for a potential protein-protein interaction event. However, a literature survey revealed that proper controls are missing in more than half of all analyzed studies. As discussed in Section 2.2.3, a negative control should be included for each tested protein-protein interaction, and the fluorescent signals should be measured quantitatively. Currently, images are presented of plant cells or tissues with a fluorescent signal, without providing a thorough quantification of the signal-to-noise ratio between the fluorescence signals from the proteins of interest and the negative controls. It is important to realize that the goal of a BiFC experiment is to obtain strong support of a protein-protein interaction and not just to obtain an image of a fluorescent cell.

The lack of reliability and robustness of the split-YFP/BiFC technology due to self-assembly of the FP halves was recognized shortly after the introduction of the method, and a plethora of modifications were suggested to overcome this problem, as discussed in Section 2.2.1. Various improvements have been suggested based on splitting the FP at different positions and the introduction of point mutations in the FP sequence, but these improvements only appeared to overcome the self-assembly problem for the tested protein combinations, or only under specific conditions. Therefore, we conclude that none of the currently used BiFC vectors is superior and that all can be used as long as the right controls are 
included and quantitative measurements are applied. Alternatively, methods based on complementation of other types of proteins, such as split-ubiquitin and split-luciferase (for review on these techniques, see [42]) can also be used. Self-assembly of protein halves is not an issue for these proteins; however, one drawback of using ubiquitin or luciferase is that due to the nature of the read-out in these systems, no information can be extracted about the subcellular position of a protein-protein interaction event. In this respect, FRET (Fluorescence Resonance Energy Transfer)-based methods are more informative than protein complementation assays, because information is obtained on both the localisation pattern of the individual proteins and of the protein-protein interaction [36,41], but these methods require sophisticated microspectroscopy equipment.

In conclusion, a low-tech, robust, and fully reliable system for the detection of protein-protein interactions in plant cells or tissues does not exist. Nonetheless, when implemented with caution split-YFP/BiFC remains a valuable tool for studying protein-protein interactions.

\section{Acknowledgments}

We thank Sander van der Krol for providing an image of an Agrobacterium infiltrated tobacco leaf shown in Figure 3.

\section{Author Contributions}

A.H. and I.A.T.N performed the experiments. R.G.H.I performed the literature study. R.G.H.I and K.B. contributed to the experimental design, and A.H., K.B. and R.G.H.I. wrote the manuscript.

\section{Conflicts of Interest}

The authors declare no conflict of interest.

\section{References}

1. Braun, P.; Aubourg, S.; van Leene, J.; de Jaeger, G.; Lurin, C. Plant Protein Interactomes. Annu. Rev. Plant Biol. 2013, 64, 161-187.

2. Immink, R.G.H.; Angenent, G.C. Transcription factors do it together: the hows and whys of studying protein-protein interactions. Trends Plant Sci. 2002, 7, 531-534.

3. Kodama, Y.; Hu, C.-D. Bimolecular fluorescence complementation (BiFC): A 5-year update and future perspectives. BioTechniques 2012, 53, 285-298.

4. Ghosh, I.; Hamilton, A.D.; Regan, L. Antiparallel leucine zipper-directed protein reassembly: Application to the green fluorescent protein. J. Am. Chem. Soc. 2000, 122, 5658-5659.

5. Hu, C.-D.; Chinenov, Y.; Kerppola, T.K. Visualization of interactions among bZIP and Rel family proteins in living cells using bimolecular fluorescence complementation. Mol. Cell 2002, 9, 789-798.

6. Bracha-Drori, K.; Shichrur, K.; Katz, A.; Oliva, M.; Angelovici, R.; Yalovsky, S.; Ohad, N. Detection of protein-protein interactions in plants using bimolecular fluorescence complementation. Plant J. 2004, 40, 419-427. 
7. Walter, M.; Chaban, C.; Schütze, K.; Batistic, O.; Weckermann, K.; Näke, C.; Blazevic, D.; Grefen, C.; Schumacher, K.; Oecking, C.; et al. Visualization of protein interactions in living plant cells using bimolecular fluorescence complementation. Plant J. 2004, 40, 428-438.

8. Kerppola, T.K. Multicolor Bimolecular Fluorescence Complementation (BiFC) Analysis of Protein Interactions with Alternative Partners. Cold Spring Harb. Protoc. 2013, 2013, doi:10.1101/pdb.top077164.

9. Lee, L.-Y.; Fang, M.-J.; Kuang, L.-Y.; Gelvin, S. Vectors for multi-color bimolecular fluorescence complementation to investigate protein-protein interactions in living plant cells. Plant Methods 2008, 4, doi:10.1186/1746-4811-4-24.

10. Waadt, R.; Schmidt, L.K.; Lohse, M.; Hashimoto, K.; Bock, R.; Kudla, J. Multicolor bimolecular fluorescence complementation reveals simultaneous formation of alternative CBL/CIPK complexes in planta. Plant J. 2008, 56, 505-516.

11. Shyu, Y.J.; Suarez, C.D.; Hu, C.-D. Visualization of ternary complexes in living cells by using a BiFC-based FRET assay. Nat. Protoc. 2008, 3, 1693-1702.

12. Shyu, Y.J.; Suarez, C.D.; Hu, C.-D. Visualization of AP-1-NF-kB ternary complexes in living cells by using a BiFC-based FRET. Proc. Natl. Acad. Sci.USA 2008, 105, 151-156.

13. Smaczniak, C.; Immink, R.G.H.; Muiño, J.M.; Blanvillain, R.; Busscher, M.; Busscher-Lange, J.; Dinh, Q.D.; Liu, S.; Westphal, A.H.; Boeren, S.; et al. Characterization of MADS-domain transcription factor complexes in Arabidopsis flower development. Proc. Natl. Acad Sci. USA 2012, 109, 1560-1565.

14. Ohashi, K.; Kiuchi, T.; Shoji, K.; Sampei, K.; Mizuno, K. Visualization of cofilin-actin and Ras-Raf interactions by bimolecular fluorescence complementation assays using a new pair of split Venus fragments. BioTechniques 2012, 52, 45-50.

15. Kodama, Y.; Hu, C.-D., An improved bimolecular fluorescence complementation assay with a high signal-to-noise ratio. BioTechniques 2010, 49, 793-805.

16. Nakagawa, C.; Inahata, K.; Nishimura, S.; Sugimoto, K. Improvement of a venus-based bimolecular fluorescence complementation assay to visualize bFos-bJun interaction in living cells. Biosci. Biotechnol. Biochem. 2011, 75, 1399-1401.

17. Kerppola, T.K. Design and implementation of bimolecular fluorescence complementation (BiFC) assays for the visualization of protein interactions in living cells. Nat. Protoc. 2006, 1 , 1278-1286.

18. Fang, Y.; Spector, D.L. BiFC imaging assay for plant protein-protein interactions. Cold Spring Harb. Protoc. 2010, 2010, doi:10.1101/pdb.prot5380.

19. Saka, Y.; Hagemann, A.I.; Smith, J.C. Visualizing protein interactions by bimolecular fluorescence complementation in Xenopus. Methods 2008, 45, 192-195.

20. PubMed-NCBI: US National Library of Medicine National Institutes of Health. Available online: http://www.ncbi.nlm.nih.gov/pubmed (accessed on 1 February 2014).

21. Lee, L.Y.; Wu, F.H.; Hsu, C.T.; Shen, S.C.; Yeh, H.Y.; Liao, D.C.; Fang, M.J.; Liu, N.T.; Yen, Y.C.; Dokladal, L.; et al. Screening a cDNA library for protein-protein interactions directly in planta. Plant Cell 2012, 24, 1746-1759.

22. Arai, R.; Ueda, H.; Kitayama, A.; Kamiya, N.; Nagamune, T. Design of the linkers which effectively separate domains of a bifunctional fusion protein. Protein Eng. 2001, 14, 529-532. 
23. Welch, D.; Hassan, H.; Blilou, I.; Immink, R.; Heidstra, R.; Scheres, B. Arabidopsis JACKDAW and MAGPIE zinc finger proteins delimit asymmetric cell division and stabilize tissue boundaries by restricting SHORT-ROOT action. Genes Dev. 2007, 21, 2196-2204.

24. Hartley, J.L.; Temple, G.F.; Brasch, M.A. DNA cloning using in vitro site-specific recombination. Genome Res. 2000, 10, 1788-1795.

25. Busso, D.; Dleagoutte-Busso, B.; Moras, D. Construction of a set gateway-based destination vectors for high-throughput cloning and expression screening in Eschericia coli. Anal. Biochem. 2005, 343, 313-321.

26. Jang, C.; Seo, E.Y.; Nam, J.; Bae, H.; Gim, Y.G.; Kim, H.G.; Cho, I.S.; Lee, Z.W.; Bauchan, G.R.; Hammond, J.; et al. Insights into alternanthera mosaic virus TGB3 functions: Interactions with nicotiana benthamiana PsbO correlate with chloroplast vesiculation and veinal necrosis caused by TGB3 over-expression. Front. Plant Sci. 2013, 4, doi:10.3389/fpls.2013.00005.

27. Park, M.-R.; Kim, K.-H. Molecular characterization of the interaction between the $N$-terminal region of Potato virus X (PVX) coat protein (CP) and Nicotiana benthamiana PVX CP-interacting protein, NbPCIP1. Virus Genes 2013, 46, 517-523.

28. Dong, C.H.; Jang, M.; Scharein, B.; Malach, A.; Rivarola, M.; Liesch, J.; Groth, G.; Hwang, I.; Chang, C. Molecular association of the Arabidopsis ETR1 ethylene receptor and a regulator of ethylene signaling, RTE1. J. Biol. Chem. 2010, 285, 40706-40713.

29. Ren, X.L.; Qi, G.N.; Feng, H.Q.; Zhao, S.; Zhao, S.S.; Wang, Y.; Wu, W.H. Calcineurin B-like protein CBL10 directly interacts with AKT1 and modulates $\mathrm{K}+$ homeostasis in Arabidopsis. Plant J. Cell Mol. Biol. 2013, 74, 258-266.

30. Merzlyak, E.M.; Goedhart, J.; Shcherbo, D.; Bulina, M.E.; Shcheglov, A.S.; Fradkov, A.F.; Gaintzeva, A.; Lukyanov, K.A.; Lukyanov, S.; Gadella, T.W.J.; et al. Bright monomeric red fluorescent protein with an extended fluorescence lifetime. Nat. Methods 2007, 4, 555-557.

31. Boutilier, K.; Offringa, R.; Sharma, V.K.; Kieft, H.; Ouellet, T.; Zhang, L.; Hattori, J.; Liu, C.-M.; van Lammeren, A.A.M.; Miki, B.L.A.; et al. Ectopic Expression of BABY BOOM Triggers a Conversion from Vegetative to Embryonic Growth. Plant Cell 2002, 14, 1737-1749.

32. Boevink, P.; McLellan, H.; Bukharova, T.; Engelhardt, S.; Birch, P. In vivo protein-protein interaction studies with BiFC: Conditions, cautions, and caveats. Methods Mol. Biol. 2014, 1127, 81-90.

33. Schutze, K.; Harter, K.; Chaban, C. Bimolecular fluorescence complementation (BiFC) to study protein-protein interactions in living plant cells. Methods Mol. Biol. 2009, 479, 189-202.

34. Waadt, R.; Kudla, J. In planta visualization of protein interactions using bimolecular fluorescence complementation (BiFC). CSH Protoc. 2008, 2008, doi:10.1101/pdb.prot4995.

35. Denecke, J.; Gossele, V.; Botterman, J.; Cornelissen, M. Quantitative analysis of transiently expressed genes in plant cells. Methods Mol. Cell. Biol. 1989, 1, 19-27.

36. Immink, R.G.H.; Gadella, T.W.J.; Ferrario, S.; Busscher, M.; Angenent, G.C. Analysis of MADS box protein-protein interactions in living plant cells. Proc. Natl. Acad. Sci.USA 2002, 99, 2416-2421.

37. Tonaco, I.A.N.; Borst, J.W.; de Vries, S.C.; Angenent, G.C.; Immink, R.G.H. In vivo imaging of MADS-box transcription factor interactions. J. Exp. Bot. 2006, 57, 33-42. 
38. Shyu, Y.J.; Liu, H.; Deng, X.; Hu, C.-D. Identification of new fluorescent protein fragments for bimolecular fluorescence complementation analysis under physiological conditions. BioTechniques 2006, 40, 61-66.

39. Berendzen, K.W.; Bohmer, M.; Wallmeroth, N.; Peter, S.; Vesic, M.; Zhou, Y.; Tiesler, F.K.E.; Schleifenbaum, F.; Harter, K. Screening for in planta protein-protein interactions combining bimolecular fluorescence complementation with flow cytometry. Plant Methods 2012, 8, doi:10.1186/1746-4811-8-25.

40. Denecke, J.; Vitale, A. The use of protoplasts to study protein synthesis and transport by the plant endomembrane system. Methods Cell Biol. 1995, 50, 335-348.

41. Bücherl, C.; Aker, J.; Vries, S.; Borst, J.W. Probing protein-protein interactions with FRET-FLIM. Methods Mol. Biol. 2010, 655, 389-399.

42. Morsy, M.; Gouthu, S.; Orchard, S.; Thorneycroft, D.; Harper, J.F.; Mittler, R.; Cushman, J.C. Charting plant interactomes: possibilities and challenges. Trends Plant Sci. 2008, 13, 183-191.

(C) 2014 by the authors; licensee MDPI, Basel, Switzerland. This article is an open access article distributed under the terms and conditions of the Creative Commons Attribution license (http://creativecommons.org/licenses/by/3.0/). 